Series A

I. MATHEMATICA

314

\title{
NOTE ON AN EQUATION IN A FINITE FIELD
}

$B Y$

VEIKKO ENNOLA

H. E L I N K I 1962

S U OMALAINEN TIEDEAKATEM IA

https://doi.org/10.5186/aasfm.1962.314 
Communicated 9 February 1962 by P. J. Myrberg and K. Inkeri 


\section{Note on an equation in a finite field}

Our intention is to use our results on the conjugacy classes of the finite unitary groups [1] in order to prove the following result. Let $\mathfrak{F}_{s}=G F\left(q^{s}\right)$ denote the finite field with $q^{s}$ elements $(q=$ power of a prime).

Theorem. Let a be $q+1$ or $q^{5}+1$. Then the number of vectors $(x, y, z)$ such that

$$
x^{a}+y^{a}+z^{a}=0,
$$

where $x, y, z$ are linearly independent elements of $\mathfrak{F}_{6}$ over $\mathfrak{F}_{2}$ equals

$$
q^{3}(q+1)^{2}(q-1)\left(q^{6}-1\right) .
$$

From this we can derive a new proof of the following fact. (For the general result see [2].)

Corollary. The number of vectors $(x, y, z)$ such that (1) is true for $a=$ $q+1$ or $q^{5}+1$ and $x, y, z$ are non-zero elements of $\mathfrak{F}_{6}$ equals

$$
\left(q^{6}-1\right)(q+1)^{2}\left(q^{4}-q^{3}+q-2\right) .
$$

Thus the statement: "In every solution of (1) with $x y z \neq 0, x, y, z$ are necessarily linearly independent over $\widetilde{F}_{2}{ }^{\prime \prime}$ is true if and only if $q=2$.

Proof of the theorem. Let $\varrho$ be a primitive element of $\mathfrak{F}_{6}$. Put $\tau=\varrho^{q^{3}-1}$ and $\tau_{i}=\tau q^{q^{i}}\left(i=0,1,2 ; \tau_{0}=\tau\right)$. Then the $\tau_{i}^{\prime}$ s are the conjugates of $\tau$ with respect to $\mathfrak{F}_{2}$ and we have

$$
\tau_{i}^{q^{3}}=\tau_{i}^{-1} .
$$

Clearly $\tau_{i} \neq \tau_{j}$ for $i \neq j$. If we write

$$
f(t)=\left(t-\tau_{0}\right)\left(t-\tau_{1}\right)\left(t-\tau_{2}\right),
$$

then $f(t)$ is an irreducible polynomial over $\mathfrak{F}_{2}$. Let $f(t)=t^{3}+a t^{2}+$ $b t+c$. Write $\tilde{f}(t)=\bar{c}^{-1}\left(\bar{c} t^{3}+\bar{b} t^{2}+\bar{a} t+1\right)$, where for $x \in \mathfrak{F}_{6}$ we put $\bar{x}=x^{q^{3}}$. (Note that if $x \in \mathfrak{F}_{2}$, then $\bar{x}=x^{q}$.) Now (2) easily implies. $f(t)=\tilde{f}(t)$. Let $M=M(f) \in G L\left(3, q^{2}\right)$ be the companion matrix of $f$. Then we know [1] that $M$ is similar to a unitary matrix and the number $\gamma(M)$ (see [1]) is 


$$
\frac{g\left(1, q^{6}\right)}{u\left(1, q^{6}\right)}=q^{3}-1 .
$$

Hence the number of non-singular matrices $X$ with elements in $\mathfrak{F}_{2}$ such that

$$
X M X^{-1} \in U\left(3, q^{2}\right)
$$

equals (see $[1]$ )

$$
\left(q^{3}-1\right) u\left(3, q^{2}\right)=q^{3}(q+1)^{2}(q-1)\left(q^{6}-1\right) .
$$

We now go up to the field $\mathfrak{F}_{6}$. We can find a matrix $P$ with elements in $\mathfrak{F}_{6}$ such that

$$
P^{-1} M P=\operatorname{diag}\left(\tau_{0}, \tau_{1}, \tau_{2}\right)=D .
$$

Since the column vectors of $P$ are eigenvectors of $M$ belonging to the distinct eigenvalues $\tau_{i}$ we may assume them to be conjugate over $\mathfrak{F}_{2}$.

Suppose now that $Y$ is a non-singular matrix with elements in $\mathfrak{F}_{6}$ such that

$$
Y D Y^{-1} \in U\left(3, q^{2}\right) \text {. }
$$

From the results of [1] it follows that $Y^{*} Y$ must be diagonal, i.e., if $Y=\left(y_{i j}\right)$, then $\sum_{i=0}^{3} y_{i j} \bar{y}_{i k}=0$, for $j \neq k$. Also, if we multiply each column vector by a suitable element of $\mathfrak{\mho}_{6}$, then we get a matrix in which the column vectors are conjugate over $\varpi_{2}$.

But this is easily seen to hold also conversely, i.e., if $Y$ satisfies these conditions, then (5) is true.

Denote by $S$ the number solutions of (1) with $a$ and $x, y, z$ as required in the theorem. Let $x, y, z$ be an arbitrary solution. Consider the matrix

$$
\left(\begin{array}{lll}
x & x_{1} & x_{2} \\
y & y_{1} & y_{2} \\
z & z_{1} & z_{2}
\end{array}\right)
$$

where $x_{i}=x^{q^{3 i}} \quad(i=1,2)$ etc. It is non-singular, because $x, y, z$ are linearly independent over $\mathfrak{F}_{2}$. If $a=q+1$, we have

$$
x \bar{x}_{2}+y \bar{y}_{2}+z \bar{z}_{2}=0
$$

and rajsing this to the $q^{5}$-th power we have

$$
x \bar{x}_{1}+y \bar{y}_{1}+z \bar{z}_{1}=0 .
$$

If $a=q^{5}+1$, we get the same results. So we see that the total number of different non-singular matrices $Y$ with conjugate column vectors satisfying $(5)$ is equal to $S$. 
Suppose now that $X$ is a non-singular matrix with elements in $\mathfrak{F}_{2}$ satisfying (3). Then $Y=X P$ is a matrix with elements in $\mathfrak{F}_{6}$ and conjugate column vectors satisfying (5).

Conversely, by using the eigenvector argument again, it is easy to see that if $Y$ is a non-singular matrix with elements in $\mathfrak{F}_{6}$ and with conjugate column vectors, then $X=Y P^{-1}$ has its elements in $\mathfrak{F}_{2}$ and satisfies (3).

Hence $S$ is equal to the expression (4) and this proves the theorem.

Proof of the Corollary Suppose that (1) is valid for $a=q+1$ or $q^{5}+1, x, y, z$ are linearly dependent over $\mathfrak{F}_{2}$ and $x y z \neq 0$. Write $u=\frac{x}{z}, v=\frac{y}{z}$. We may assume that $a=q+1$, otherwise we raise (1) to the $q$-th power. We have

$$
u^{q+1}+v^{q+1}+1=0
$$

where $u, v, 1$ are linearly dependent over $\mathfrak{F}_{2}$. Clearly we may assume

$$
u=b v+c, \quad b, c \in \mathfrak{F}_{2} .
$$

'Then

$$
u^{q}=\bar{b} v^{q}+\bar{c}
$$

Multiplying these, using (6), and then raising the equation to the power $q, q^{2}, q^{3}$, respectively, we get the following system of equations

$$
\left\{\begin{array}{l}
(b \bar{b}+1) v^{q+1}+\bar{b} c v^{q}+b \bar{c} v+c \bar{c}+1=0 \\
(b \bar{b}+1) v^{q^{2}+q}+\bar{b} c v^{q}+b \bar{c} v^{q^{2}}+c \bar{c}+1=0 \\
(b \bar{b}+1) v^{q^{3}+q^{2}}+\bar{b} c v^{q^{3}}+b \bar{c} v^{q^{2}}+c \bar{c}+1=0 \\
(b \bar{b}+1) v^{q^{4}+q^{3}}+\bar{b} c v^{q^{3}}+b \bar{c} v^{q^{4}}+c \bar{c}+1=0 .
\end{array}\right.
$$

Consider here $\bar{b} b+1, \bar{b} c, b \bar{c}, \bar{c} \bar{c}-1$ as indeterminates. Then the determinant of $(7)$ is

$$
v^{q^{2}+2 q+1}\left(v^{q^{2}-1}-1\right)\left(v^{q^{4}-q^{2}}-1\right)\left(v^{q^{3}-q}-1\right)^{2} .
$$

If $v \notin \mathfrak{F}_{2}$, then $v^{q^{2}-1}-1 \neq 0$ and thus (8) does not vanish. Hence $b \bar{b}+1=c \bar{c}+1=b \bar{c}=0$, which is impossible. So we must have $u$, $v \in \mathfrak{F}_{2}$

Write (6) in the form

$$
u^{q+1}=-\left(1+v^{q+1}\right) .
$$

We can take $v$ to be any one of the $q^{2}-1$ elements of $\mathfrak{F}_{2}$ except those $q+1$ which make $v^{q+1}=-1$. For each $v$ we have $q+1$ possible $u$ 's. 
So the total number of linearly dependent solutions of $(6)$ is $(q+1)^{2}$ $(q-2)$. Multiplying this by $q^{6}-1$ we get the corresponding number for the equation (1). From this the Corollary follows.

\section{References}

[1] Ennola, Veikno: On the conjugacy classes of the finite unitary groups. - Ann. Acad. Sci. Fenn. A I, No. 313 (1962).

[2] Mitchell, Howard H.: On the congruence $c x^{i}+1 \equiv d y^{i}$ in a Galois field. . Ann. of Math. 18.3 (1917), pp. 120-131.

University of Turku

Finland

Printed April 1962. 